MRI-P-020704

hep-ph/0207247

\title{
QCD Corrections to Resonant Slepton Production in Hadron Colliders
}

\author{
Debajyoti Choudhury, Swapan Majhi and V. Ravindran \\ Harish-Chandra Research Institute, Chhatnag Road, Jhusi, Allahabad 211 019, India \\ E-mail: debchou,swapan,ravindra@mri.ernet.in
}

\begin{abstract}
We consider resonant production of sneutrino and slepton at hadronic colliders such as the Tevatron and the LHC within the context of a $R$-parity violating supersymmetric model. We present next to leading order QCD corrections to total cross sections which originate from both quark- as well as gluon-initiated processes. For couplings involving only the first generation quarks, the $K$ factor at the Tevatron can be as large as 1.5 for a $100 \mathrm{GeV}$ sfermion and falls to nearly 1.1 as the sfermion mass reaches $1 \mathrm{TeV}$. At the LHC, the variation is between 1.2 and 1.45 for masses less than $2 \mathrm{TeV}$. While the dependence on the parton density parametrization is found to be mild, this ceases to be true if the strange quark plays a dominant role in the production process. We also study the renormalization and factorizationscale dependence and find it to be less pronounced for the NLO cros sections as compared to the LO. The results obtained in this article are also applicable to resonant production of any color-neutral scalar.
\end{abstract}




\section{Introduction}

The scalar sector of the Standard Model (SM), while being integral to the validity of this otherwise eminently successful model, has also been somewhat of an embarrassment. Quite apart from the fact that the Higgs particle has, till date, defied all attempts at detecting it, there is the theoretical problem that the mass of this particle is not protected by any symmetry (at least, not within the SM). Consequently, quantum corrections would tend to drive it to the next higher scale of interaction, an eventuality that, apart from running counter to the indications coming from electroweak precision measurements [1], would also lead to a loss of perturbative unitarity. To overcome this as well as certain other lacunae of the SM, many models going beyond the SM have been proposed. Two of the most attractive classes of such models comprise those incorporating supersymmetry [2] and/or grand unification [3] (especially scenarios with a low intermediate scale [4]). Such models, however, predict, additional particle states, including scalars. What is most interesting is that the coupling of the first generation SM fermions to these scalars need no longer be suppressed, thus offering hope for novel signatures.

The last-mentioned feature has, naturally, attracted much attention, especially in the context of the current and future colliders. Apart from pair-production (determined, in the most part, by the gauge interactions), an enhanced coupling to fermions opens up the possibility of resonance production at colliders whether hadronic $(p \bar{p}$ or $p p)$ [5] [7], $e^{+} e^{-}$[8, 9] or $e^{ \pm} p$ [10]. These studies conclude that not only is discovery guaranteed for a significantly large part of the parameter space, even a measurement of the coupling strengths to a reasonable degree of accuracy might be possible.

Most of these analyses, however, have been performed only at the Born level. In view of the interesting consequences, it is desirable that quantum corrections to such resonance production processes should be investigated. While this has been done in the context of ep colliders [11], a similar exercise has not been attempted for hadronic colliders. In this paper, we seek to rectify this lacuna. Before we embark on such a venture, however, it is important to note that, in a generic model going beyond the SM, scalars could appear in many a hue. The quantum numbers are of crucial importance as these would determine not only the production cross-sections but also the dominant decay channels and hence the possible modes of discovery. For the sake of concreteness, we shall confine ourselves to a discussion of sneutrino and slepton production in the context of a $R$-parity violating supersymmetric model. While, at first, this may seem to be a very restrictive assumption, in reality, these constitute very typical examples of colour-neutral scalars. One might as well have considered a generic multi-higgs model wherein some of the low-lying states have an enhanced coupling with the lighter fermions. The prime rationale behind our choice is that while the scalar masses can be protected naturally in supersymmetric mod-

els, the same is not so straightforward in non-supersymmetric models (grand unified or otherwise). Moreover, the $R$-parity violating Minimal Supersymmetric Standard Model (MSSM) being a richer (low-energy) theory, offers a larger set of possibilities, both in the context of the neutrino anomalies seen at KAMIOKANDE [12] or KARMEN [13] or the unexplained high- $Q^{2}$ events at HERA [10]. 
The plan of the paper is as follows. We start this article (Section 2) with a brief review of the status of $R$-parity conservation within the MSSM.. Section 3 describes the particular resonance production processes (at the lowest-order) that we are interested in. The formalism and the calculations for the NLO corrections are set out in the following section. In section of, we present the numerical results and a discussion thereof. And finally we summarise.

\section{$2 \quad R$-parity violation: a mini-review}

As is well known, within the SM, both baryon $(B)$ and lepton $(L)$ number conservation are but accidental consequences of the choice of the particle content' In extensions of the SM, such an accidental occurrence is obviously not guaranteed. For example, in a generic grand unified theory (GUT), both the gauge and the scalar sector interactions violate each of $B$ and $L$. This is potentially catastrophic as a simultaneous breaking of both $B$ and $L$ could lead to rapid proton decay. Within GUTs, however, gauge boson-mediated proton decay is naturally suppressed on account of the large gauge-boson masses. On the other hand, the scalar sector has to be carefully chosen so as to suppress any effective operator leading to proton decay.

Within the context of the MSSM though, we do not have the option of demanding the sfermion or gaugino fields to be superheavy. However, a similar suppression can be achieved by introducing a discrete symmetry, $R \equiv(-1)^{3(B-L)+2 S}$ (with $S$ denoting the spin of the field) 14 that serves to rule out both $B$ and $L$ violating terms in the superpotential. In addition, this symmetry renders the lightest supersymmetric partner absolutely stable. The introduction of this symmetry is clearly an ad hoc measure and is not even strictly essential to rule out proton decay. Hence, it is of interest to consider possible violations of this symmetry especially since it has rather important experimental consequences, not the least of which concerns the detection of the supersymmetric partners.

The possible $R$-parity violating $\left(R_{\mathrm{p}}\right)$ terms in the superpotential can be parametrised as

$$
\mathcal{W}_{R_{p}}=\mu_{i} L_{i} H_{2}+\lambda_{i j k} L_{i} L_{j} E_{k}^{c}+\lambda_{i j k}^{\prime} L_{i} Q_{j} D_{k}^{c}+\lambda_{i j k}^{\prime \prime} U_{i}^{c} D_{j}^{c} D_{k}^{c},
$$

where $L_{i}$ and $Q_{i}$ are the $S U(2)$-doublet lepton and quark superfields, $E_{i}^{c}, U_{i}^{c}, D_{i}^{c}$ the singlet superfields and $H_{i}$ the Higgs superfields. Clearly $\lambda_{i j k}$ is antisymmetric under the interchange of the first two indices, while $\lambda_{i j k}^{\prime \prime}$ is antisymmetric under the interchange of the last two. Whereas the first three terms in eqn.(11) violate $L$, the last term falls foul of $B$ conservation. To circumvent the constraints imposed by the non-observance of proton decay, we, thus, need to have at least one of the two sets of couplings to be vanishingly small. For the purpose of this paper, we assume that $B$ is a good symmetry of the theory, or in other words all of $\lambda_{i j k}^{\prime \prime}$ are zero. This has the added advantage that all dimension six operators leading to proton decay are suppressed [15] along with the dimension five ones. Such a scenario might be motivated within certain theoretical

\footnotetext{
${ }^{1}$ Indeed, non-perturbative effects within the SM itself do break $B+L$ symmetry.
} 
frameworks [15, 16] and also renders simpler the problem of preservation of GUT-scale baryon asymmetry [17]. Although the presence of the other $R_{\mathrm{p}}$ terms could, in principle, affect the baryon asymmetry of the universe, such bounds are highly model-dependent and can be evaded [18]. For example, in cases where at least one $L$-violating coupling involving a particular lepton family is small enough $\left(\lesssim 10^{-7}\right)$ so as to (almost) conserve the corresponding lepton flavour over cosmological time scales, such bounds are no longer effective.

Each of the terms in eqn.(可) has its unique set of consequences, whether in low-energy phenomenology or in resonance production. For example, while $\lambda_{i j k}$ lead to resonant sneutrino production in $e^{+} e^{-}$collider [8,9], a non-zero $\lambda_{i j k}^{\prime \prime}$ leads to resonant squark production in hadron-hadron collisions [5, [7]. Even richer phenomenology is associated with the $\lambda_{i j k}^{\prime}$ terms. For example, the exchange of a $t$-channel sfermion alters significantly [19] both the rates and the kinematic distributions of processes such as $\bar{t} t$ production or Drell-Yan production of dileptons. More strikingly, a non-zero $\lambda_{i j k}^{\prime}$ can lead to both resonant squark production at an $e^{ \pm} p$ facility [10] as well as to resonant slepton and sneutrino production at a hadronic collider [5, [6]. It is this last aspect that we shall concentrate on.

The relevant part of the Lagrangian can be written in terms of the component fields as

$$
\begin{aligned}
\mathcal{L}_{\lambda^{\prime}}=\lambda_{i j k}^{\prime} & {\left[\overline{d_{k R}} \nu_{i L} \tilde{d}_{j L}+\overline{d_{k R}} d_{j L} \tilde{\nu}_{i L}+\overline{\left(\nu_{i L}\right)^{c}} d_{j L} \tilde{d}_{k R}^{*}\right.} \\
& \left.-\overline{d_{k R}} \ell_{i L} \tilde{u}_{j L}-\overline{d_{k R}} u_{j L} \tilde{\ell}_{i L}-\overline{\left(\ell_{i L}\right)^{c}} u_{j L} \tilde{d}_{k R}^{*}\right]+ \text { h.c. }
\end{aligned}
$$

Thus, while the squarks behave as leptoquarks in a non-supersymmetric theory, the sleptons/sneutrinos behave as if they are charged/neutral Higgses in a multi-Higgs-doublet scenario. Clearly, non-zero values for these couplings could lead to rather striking phenomenological consequences. For example, pair production of squarks that subsequently decay through an $L$ violating interaction, leads to a final state comprising a dilepton pair along with jets [20]. More interestingly, the gluino production cross-section is larger and, in addition, can lead to like-sign dileptons, thereby making the signal stand out even more [21]. Non-observation of such signals thus rules out a relatively light squark or gluino along with a sizable $\lambda^{\prime}$. However, these analyses can say very little about sleptons/sneutrinos as the corresponding production cross-sections are much smaller than those for a squark/gluino. At an $e^{+} e^{-}$collider though, both pair production of sleptons/sneutrinos and the corresponding backgrounds are weak processes and hence such colliders are expected to be better suited for this particular quest. Unfortunately, an $e^{+} e^{-}$ collider energetic enough to pair-produce sleptons is still very much in the future.

We now turn to the constraints from low-energy phenomenology. Non-zero $\lambda^{\prime}$ s can lead, for example, to additional four-fermi operators that may contribute to meson decays, neutral meson mixings, some of which may be forbidden otherwise. Since the absence of tree-level flavour changing neutral current processes lead to rather severe constraints on the simultaneous presence of more than one $\lambda^{\prime}$ [22], we shall henceforth restrict ourselves to only one non-zero $\lambda^{\prime}$. In Table [1, we list the currently known bounds on several of these couplings]. The strongest bound is on $\lambda_{111}^{\prime}$ and is derived from non-observation

\footnotetext{
${ }^{2} \mathrm{~A}$ more complete list can be found in refs. [23].
} 
of neutrinoless double beta decay (a) 24]. The others are much weaker and are derived from $(b)$ upper bound on the mass of the $\nu_{e}$ [16, 25 27]; data on $(c)$ charged-current universality [9]; $(d)$ atomic parity violation [28]; $(e) \tau \rightarrow \pi \nu_{\tau}$ and $D \rightarrow K l \nu$ [26]; and $(f)$ $D^{0}-\overline{D^{0}}$ mixing [22]. Since these bounds are derived from effective 4-fermi operators, they

\begin{tabular}{||c|c||c|c||c|c||}
\hline$\{i j k\}$ & Existing bounds & $\{i j k\}$ & Existing bounds & $\{i j k\}$ & Existing bounds \\
\hline 111 & $0.001^{a)}$ & 211 & $0.09^{c)}$ & 311 & $0.10^{e)}$ \\
112 & $0.02^{c)}$ & 212 & $0.09^{c)}$ & 312 & $0.10^{e)}$ \\
121 & $0.035^{d)}$ & 221 & $0.18^{e)}$ & 321 & $0.20^{f)}$ \\
122 & $0.02^{b)}$ & 222 & $0.18^{e)}$ & 322 & $0.20^{f)}$ \\
\hline
\end{tabular}

Table 1: The upper bounds on the $\lambda^{\prime}$-type $R_{\mathrm{p}}$ couplings of interest for a common sfermion mass $\tilde{m}=100 \mathrm{GeV}$. The superscripts refer to the specific experiments leading to the constraints and as described in the text.

typically scale like the mass of the exchanged sfermion?. Two points may be noted here. First, many of these bounds are actually applicable only to particular combinations of couplings and masses and reduce to those in the table only under the assumption of only one coupling being non-zero. And secondly, in meson decays, most often it is the squark that is exchanged; hence sleptons/sneutrinos could very well be much lighter without contradicting the bounds.

\section{Leading order cross-section}

The $R_{\mathrm{p}}$ interaction Lagrangian, as presented in eqn.(2), allows for the following resonance production processes at a hadronic collider:

$$
\begin{aligned}
& \lambda_{i j k}^{\prime}: d_{j}+\bar{d}_{k} \rightarrow \tilde{\nu}_{i}, \quad d_{k}+\bar{d}_{j} \rightarrow \tilde{\nu}_{i}^{*} \\
& : \quad d_{k}+\bar{u}_{j} \rightarrow \tilde{\ell}_{i}, \quad u_{j}+\bar{d}_{k} \rightarrow \tilde{\ell}_{i}^{*} \cdot
\end{aligned}
$$

The conjugate processes obviously have identical cross-sections at the Tevatron, though not at the LHC.

Before we start, we will make a few simplifying assumptions. Since QCD is flavourblind, the form of the strong interaction corrections would be independent of the particular initial state quark in eqn.(3). Hence, for the sake of simplicity, we choose to develop the formalism for the case of identical quarks. Furthermore, rather than consider a chiral coupling to the scalars, we assume that the interaction is purely a scalar one. The chirality structure can be accounted for at a later stage simply by introducing an extra factor of $1 / 2$. Note that neither of these assumptions imply a loss of generality.

\footnotetext{
${ }^{3}$ Of those listed in Table 1, the only exceptions to this rule are the bounds for $\lambda_{111}^{\prime}$ and $\lambda_{122}^{\prime}$ [16, 24, 26].
} 
The leading order process of interest is then

$$
\bar{q}\left(p^{\prime}\right)+q(p) \rightarrow S(q)
$$

where $p$ and $p^{\prime}$ are the momenta of incoming quark and anti-quark respectively and $q$ that of the outgoing scalar. The amplitude for this process is given by

$$
M^{(0)}=-i \lambda \bar{v}\left(p^{\prime}\right) u(p)
$$

where $\lambda$ is the scalar coupling constant. Since we consider only light quarks in the initial state, we have

$$
\left(p^{\prime}\right)^{2}=p^{2}=0
$$

whereas the scalar has a (large) mass $m_{q}$. The cross-section for the process in (eqn. (1) is

$$
\sigma_{0}=\frac{1}{3} \frac{1}{4\left(p \cdot p^{\prime}\right)} \int \frac{d^{n-1} q}{(2 \pi)^{n-1} 2 q_{0}} \frac{1}{4}\left|M^{(0)}\right|^{2}(2 \pi)^{n} \delta^{n}\left(p^{\prime}+p-q\right)
$$

where the factor $1 / 4$ arises from the spin averaging for the incoming quarks and $4 p \cdot p^{\prime}$ is the flux factor. Taking the space-time dimension $n=4$, the above reduces to

$$
\sigma_{0}=\lambda^{2} \frac{1}{3} \frac{\pi}{2 s} \delta(1-\tau)
$$

where

$$
\tau=\frac{m_{q}^{2}}{s}, \quad s=\left(p^{\prime}+p\right)^{2}, \quad m_{q}^{2}=q \cdot q
$$

\section{$4 \quad$ NLO corrections}

The QCD correction to the process of interest has contributions from two different, but related, sources. First, the quark-pair-initiated process itself receives radiative correction. To this must be added the contribution arising from radiating off a soft gluon. And secondly, since our true initial state is not quarks, but (anti-)protons, we must include possible contributions from "initial-state" gluons as well. We consider each in turn.

\subsection{Correction to the $q \bar{q}$ initiated process}

To calculate the QCD radiative correction to this process, we start by computing the $\mathcal{O}\left(g_{s}^{2}\right)$ corrections to the vertex function and the self energy, where $g_{s}$ is the QCD strong coupling constant. A prime ingredient for this is the calculation of the corresponding renormalisation constants $Z_{\lambda}$ and $Z_{2}$. Even on regulating the ultraviolet (UV) divergences, we would, expectedly, be left with infra-red (IR) divergences, part of which will be cancelled once we take into account the soft gluon bremsstrahlung contribution. Throughout our

calculation we shall use dimensional regularisation to regulate any divergence and the $\overline{M S}$ prescription for renormalising the results. 
Let us first consider the vertex function $M^{V}$ upto order $g_{s}^{2}$. This can be expanded as

$$
M^{V}=M^{(0)}+M^{V(1)}
$$

where $M^{(0)}=-i \lambda \bar{v}\left(p^{\prime}\right) u(p)$, and

$$
M^{V(1)}=-\lambda g_{s}^{2} \frac{1}{\left(\mu^{2}\right)^{\frac{n}{2}-2}} \int \frac{d^{n} k}{(2 \pi)^{n}} \frac{\bar{v}\left(p^{\prime}\right) t^{a} t^{a}\left[\gamma^{\mu}\left(-\not p^{\prime}+\not k\right)(\not p+\not k) \gamma_{\mu}\right] u(p)}{\left[\left(p^{\prime}-k\right)^{2}+i \eta\right]\left[(p+k)^{2}+i \eta\right]\left[k^{2}+i \eta\right]} .
$$

The introduction of the (arbitrary) mass scale $\mu$ (called renormalisation scale) is necessary to render the strong coupling constant $g_{s}$ dimensionless in $n$ space-time dimensions. The matrices $t^{a}$ are the Gell-Man matrices and satisfy $\left(t^{a} t^{a}\right)_{i j}=C_{F} \delta_{i j}$.

On using the equations of motion, the above can be simplified to

$$
M^{V(1)}=\bar{v}\left(p^{\prime}\right)(-i \lambda) \bar{\Gamma}^{(1)} u(p),
$$

where

$$
\bar{\Gamma}^{(1)}=-i g_{s}^{2} C_{F} \frac{1}{\left(\mu^{2}\right)^{\frac{n}{2}-2}}\left[-2 q^{2} I_{1}+4\left(p-p^{\prime}\right)^{\mu} I_{\mu}+n g^{\mu \nu} I_{\mu \nu}\right],
$$

with

$$
I_{\{1, \mu, \mu \nu\}}=\int \frac{d^{n} k}{(2 \pi)^{n}} \frac{\left\{1, k_{\mu}, k_{\mu} k_{\nu}\right\}}{\left[\left(p^{\prime}-k\right)^{2}+i \eta\right]\left[(p+k)^{2}+i \eta\right]\left[k^{2}+i \eta\right]} .
$$

Naive power counting shows that $I_{\mu \nu}$ is logarithmically divergent in 4-dimensions, while the other two are convergent. The integrals can be evaluated explicitly (for example, using Feynman parametrisation) and the results expressed in terms of Gamma functions. The resultant vertex function is then $(\epsilon \equiv n-4)$

$$
\bar{\Gamma}^{(1)}=\frac{\alpha_{s}}{4 \pi} C_{F}\left(\frac{-q^{2}}{4 \pi \mu^{2}}\right)^{\frac{\epsilon}{2}} \frac{\Gamma^{2}\left(1+\frac{\epsilon}{2}\right) \Gamma\left(1-\frac{\epsilon}{2}\right)}{\Gamma(2+\epsilon)}\left[-\frac{2}{\epsilon^{2}}(2+\epsilon)^{2}\right] .
$$

The renormalisation constant $Z_{\lambda}$ is defined through the relation

$$
Z_{\lambda}^{-1}=1+\left.\bar{\Gamma}^{(1)}\right|_{U V}
$$

and, of course, depends on the way the ultraviolet divergent part $\left.\Gamma^{(1)}\right|_{U V}$ is isolated. Within the $\overline{M S}$ scheme, it can easily be ascertained to be

$$
Z_{\lambda}=1+\frac{\alpha_{s}}{4 \pi \Gamma(1+\epsilon / 2)} C_{F}\left(\frac{1}{4 \pi}\right)^{\frac{\epsilon}{2}}\left(\frac{8}{\epsilon}\right)
$$

with $\alpha_{s} \equiv g_{s}^{2} / 4 \pi$.

The self energy correction to the Born amplitude (say, to the quark only) can be expressed as

$$
M^{S}=-i \lambda \bar{v}\left(p^{\prime}\right) \frac{1}{p} \bar{\Sigma}(\not p) u(p)
$$


where

$$
\bar{\Sigma}(\not p)=-i g_{s}^{2} \frac{C_{F}}{\left(\mu^{2}\right)^{\frac{n}{2}-2}} \int \frac{d^{n} k}{(2 \pi)^{n}} \frac{\gamma^{\mu}(\not p+\not k) \gamma_{\mu}}{\left[k^{2}+i \eta\right]\left[(p+k)^{2}+i \eta\right]} .
$$

Notice that $\Sigma(\not p)$ does not contribute to the amplitude given in eqn.(4) due to the massless nature of the light quarks. On the other hand, the above equation can be used to determine the wave function renormalisation constant $Z_{2}$ through the relation

$$
\left.\frac{d \bar{\Sigma}(\not p)}{d \not p}\right|_{\not p=0}=i\left(Z_{2}^{-1}-1\right)
$$

In the $\overline{M S}$ scheme (and in a scale independent way) this can be rewritten as

$$
Z_{2}=1+\frac{\alpha_{s}}{4 \pi \Gamma(1+\epsilon / 2)} C_{F}\left(\frac{1}{4 \pi}\right)^{\frac{\epsilon}{2}}\left(\frac{2}{\epsilon}\right)
$$

Our next task is to compute the virtual contributions to the process given in eqn.(4). In order to do this, we have to redefine the fields and the coupling constants in terms of the renormalised ones (and, of course, the renormalisation constants $Z_{2}$ and $Z_{\lambda}$.) This is equivalent to adding UV counter terms corresponding to the vertex function and self energy contribution. Note that self energy contribution to the amplitude is identically zero due to the on-shell condition. Hence only vertex function and the counter terms contribute to the amplitude, and

$$
M^{(0)+v i r+C T}=M^{(0)}+M^{V(1)}+M^{C T} .
$$

It turns out that the effect of the counter term(CT) is

$$
M^{(0)}+M^{C T}=\left(-i \lambda_{R}\right) \frac{Z_{\lambda}}{Z_{2}} \bar{v}\left(p^{\prime}\right) u(p)
$$

where the renormalised coupling $\lambda_{R}$ is related to the unrenormalised one through

$$
\lambda_{R}=\lambda \frac{Z_{2}}{Z_{\lambda}} .
$$

The virtual and counter term contribution to the Born diagram can be expressed as

$$
\sigma^{(0)+v i r+C T}=\frac{1}{3} \frac{1}{4\left(p \cdot p^{\prime}\right)} \int \frac{d^{n-1} q}{(2 \pi)^{n-1} 2 q_{0}} \frac{1}{4}\left|M^{(0)+v i r+C T}\right|^{2}(2 \pi)^{n} \delta^{n}\left(p+p^{\prime}-q\right) .
$$

Substituting eqns.(17,21) in eqn.(25), we have

$$
\sigma^{(0)+v i r+C T}=\frac{1}{3} \frac{\pi}{2 s} \lambda_{R}^{2}\left(1+\frac{\alpha_{s}}{4 \pi} C_{F} F^{(1)}\right) \delta(1-\tau)
$$

where

$$
F^{(1)}=\frac{4 \pi}{\alpha_{s} C_{F}}\left(\frac{Z_{\lambda}^{2}}{Z_{2}^{2}}+2 \bar{\Gamma}^{(1)}-1\right)
$$


Expanding around $\epsilon=0$ and neglecting those terms which vanish in the limit $\epsilon \rightarrow 0$ we get

$$
F^{(1)}=\frac{2}{\Gamma\left(1+\frac{\epsilon}{2}\right)}\left(\frac{1}{4 \pi}\right)^{\frac{\epsilon}{2}}\left[-\frac{8}{\epsilon^{2}}+\frac{6}{\epsilon}-\frac{4}{\epsilon} \ln \left(\frac{q^{2}}{\mu^{2}}\right)-\ln ^{2}\left(\frac{q^{2}}{\mu^{2}}\right)-2+\pi^{2}\right]
$$

Next, we compute the contribution from the gluon bremsstrahlung to $\mathcal{O}\left(\alpha_{s}\right)$ :

$$
\bar{q}\left(p^{\prime}\right)+q(p) \rightarrow S(q)+g(k) .
$$

Here, $k$ is the momentum of the out going gluon. The corresponding cross-section is

$$
\sigma_{r}=\frac{1}{3} \frac{1}{4\left(p \cdot p^{\prime}\right)} \int d P S_{2} \frac{1}{4}\left|M_{r}\right|^{2}
$$

where the two body phase space is

$$
d P S_{2}=\frac{d^{n-1} k}{(2 \pi)^{n-1} 2 k^{0}} \frac{d^{n-1} q}{(2 \pi)^{n-1} 2 q^{0}}(2 \pi)^{n} \delta^{n}\left(k+q-p-p^{\prime}\right)
$$

and the amplitude $M_{r}$ is given by

$$
M_{r}=-\lambda g_{s} \bar{v}\left(p^{\prime}\right) t^{a}\left[\frac{\gamma^{\mu}\left(\not k-\not p^{\prime}\right)}{\left(k-p^{\prime}\right)^{2}}+\frac{(\not p-\not k) \gamma^{\mu}}{(p-k)^{2}}\right] u(p) \varepsilon_{\mu}^{*}(k) .
$$

The phase space integration can be easily done in the centre of mass frame of incoming quarks wherein the momenta of the particles can be parametrised as

$$
\begin{aligned}
p & =\frac{\sqrt{s}}{2}(1,0,0,1), & p^{\prime} & =\frac{\sqrt{s}}{2}(1,0,0,-1) \\
k & =|q|(1, \sin \theta, 0, \cos \theta), & q & =\left(q_{0},-|q| \sin \theta, 0,-|q| \cos \theta\right) \\
q_{0} & =\frac{\sqrt{s}}{2}(1+\tau) & |q| & =\frac{\sqrt{s}}{2}(1-\tau),
\end{aligned}
$$

with the square of the matrix element being given by

$$
\left|M_{r}\right|^{2}=4 \lambda^{2} g_{s}^{2} C_{F}\left(n-2-\frac{4}{1-\tau}+\frac{4}{(1-\tau)^{2}}\right)\left(\frac{1}{1+y}+\frac{1}{1-y}\right) .
$$

Substituting the above in eqn.(34) and performing the integration, we have

$$
\begin{aligned}
\sigma_{r}= & \frac{1}{3} \frac{\alpha_{s}}{4 s} C_{F} \lambda^{2} \frac{(4 \pi)^{-\epsilon / 2}}{\Gamma\left(1+\frac{\epsilon}{2}\right)}\left[\left(\frac{8}{\epsilon^{2}}+\frac{4}{\epsilon} \ln \left(\frac{q^{2}}{\mu^{2}}\right)+\ln ^{2}\left(\frac{q^{2}}{\mu^{2}}\right)-\frac{\pi^{2}}{3}\right) \delta(1-\tau)\right. \\
& +\frac{4}{\epsilon} \frac{1+\tau^{2}}{(1-\tau)_{+}}+2(1-\tau)+4\left(1+\tau^{2}\right)\left(\frac{\ln (1-\tau)}{(1-\tau)}\right)_{+} \\
& \left.+2 \frac{1+\tau^{2}}{(1-\tau)_{+}} \ln \left(\frac{q^{2}}{\mu^{2}}\right)-2 \frac{1+\tau^{2}}{(1-\tau)} \ln (\tau)\right]
\end{aligned}
$$


In the above, the "+" prescription for a function $f(z)$ is defined as

$$
\int_{0}^{1} d z \frac{f(z)}{(1-z)_{+}}=\int_{0}^{1} d z \frac{f(z)-f(1)}{(1-z)} .
$$

Notice that the above integral is divergent as $z \rightarrow 1$. This is but a manifestation of the collinear divergence arising due to the masslessness of the incoming quarks, and can be safely absorbed into unrenormalised parton densities by a suitable counter term. Within the $\overline{M S}$ scheme, the said counter term is given by

$$
\sigma^{c t}=\frac{1}{3} \frac{\alpha_{s}}{4 s} C_{F} \lambda_{R}^{2} \frac{1}{\Gamma\left(1+\frac{\epsilon}{2}\right)}\left(\frac{M^{2}}{4 \pi \mu^{2}}\right)^{\frac{\epsilon}{2}}\left[-6 \delta(1-\tau)-\frac{4}{\epsilon} \frac{1+\tau^{2}}{(1-\tau)_{+}}\right],
$$

where $M$ is the factorisation scale. Adding the virtual corrections to the bremsstrahlung contribution with the collinear counter term (eqn.(37)), we get, upto $\mathcal{O}\left(\alpha_{s}\right)$,

$$
\begin{aligned}
\sigma^{q}= & \frac{1}{3} \frac{\pi}{2 s} \lambda_{R}^{2} \delta(1-\tau)+\frac{1}{3} \frac{\alpha_{s}}{4 s} C_{F} \lambda_{R}^{2}\left[\left(-3 \ln \left(\frac{q^{2}}{\mu^{2}}\right)-2+\frac{2 \pi^{2}}{3}\right) \delta(1-\tau)\right. \\
& +3 \delta(1-\tau) \ln \left(\frac{q^{2}}{M^{2}}\right)+2(1-\tau) \\
& \left.+4\left(1+\tau^{2}\right)\left(\frac{\ln (1-\tau)}{(1-\tau)}\right)_{+}+2 \frac{1+\tau^{2}}{(1-\tau)_{+}} \ln \left(\frac{q^{2}}{M^{2} \tau}\right)\right] .
\end{aligned}
$$

\subsection{Contribution from the gluon initiated process}

We now compute the final piece, namely the contribution of the Compton-like process to order $\alpha_{s}$ :

$$
g(k)+q(p) \rightarrow S(q)+q\left(p^{\prime}\right) .
$$

The cross-section is given by

$$
\sigma_{c}=\frac{1}{4(p \cdot k)} \frac{1}{8} \frac{1}{3} \int d P S_{2} \frac{1}{2(n-2)}\left|M_{c}\right|^{2}
$$

with the amplitude $M_{c}$ being

$$
M_{c}=-\lambda g_{s} \bar{u}\left(p^{\prime}\right) t^{a}\left[\frac{\gamma^{\mu}\left(\not p^{\prime}-\not k\right)}{\left(p^{\prime}-k\right)^{2}}+\frac{(\not p+\not k) \gamma^{\mu}}{(p+k)^{2}}\right] u(p) \varepsilon_{\mu}(k) .
$$

Once again, the two-body phase space $\left(d P S_{2}\right)$ integration can be easily done in the centre of mass frame of incoming gluon and quark wherein

$$
\left|M_{c}\right|^{2}=4 \lambda^{2} g_{s}^{2}\left[\left(-4 \tau+\frac{(n-2)}{1-\tau}\right) \frac{4}{1+y}+y(1-\tau)(n-2)+6+10 \tau-3 n-n \tau\right] \text {. }
$$


On performing the angular integration, we get

$$
\begin{aligned}
\sigma_{c}= & \frac{\lambda^{2} g_{s}^{2}}{16 \pi s} \frac{1}{3}\left(\frac{q^{2}}{4 \pi \mu^{2} \tau}\right)^{\frac{\epsilon}{2}} \frac{\Gamma\left(1+\frac{\epsilon}{2}\right)}{\Gamma(1+\epsilon)} \frac{1}{4 \epsilon(2+\epsilon)} \\
& \times \frac{1}{(1-\tau)^{-\epsilon}}\left[8\left(\tau^{2}+(1-\tau)^{2}\right)+4 \epsilon-\frac{\epsilon(1-\tau)}{1+\epsilon}(6(1-\tau)+\epsilon(3+\tau))\right] \\
= & \frac{\alpha_{s}}{4 s} \frac{1}{3} \lambda^{2} \frac{1}{\Gamma\left(1+\frac{\epsilon}{2}\right)}\left(\frac{1}{4 \pi}\right)^{\frac{\epsilon}{2}}\left[\frac{1}{\epsilon}\left(\tau^{2}+(1-\tau)^{2}\right)\right. \\
& +\frac{1}{2}\left(\tau^{2}+(1-\tau)^{2}\right) \ln \left(\frac{q^{2}}{\mu^{2}}\right)+\frac{1}{2}\left(\tau^{2}+(1-\tau)^{2}\right) \ln \left(\frac{(1-\tau)^{2}}{\tau}\right) \\
& \left.+\frac{1}{4}(1-\tau)(7 \tau-3)\right] .
\end{aligned}
$$

With the $\overline{M S}$ counter term to remove the collinear divergence coming from the massless incoming gluon and quark being given by

$$
\sigma^{c t}=\frac{\alpha_{s}}{4 s} \frac{1}{3} \lambda_{R}^{2} \frac{1}{\Gamma\left(1+\frac{\epsilon}{2}\right)}\left(\frac{M^{2}}{\mu^{2}}\right)^{\frac{\epsilon}{2}}\left(\frac{1}{4 \pi}\right)^{\frac{\epsilon}{2}}\left[-\frac{1}{\epsilon}\left(\tau^{2}+(1-\tau)^{2}\right)\right],
$$

we finally have, to $\mathcal{O}\left(\alpha_{s}\right)$,

$$
\begin{aligned}
\sigma^{g}= & \frac{\alpha_{s}}{4 s} \frac{1}{3} \lambda_{R}^{2}\left[\frac{1}{2}\left(\tau^{2}+(1-\tau)^{2}\right) \ln \left(\frac{q^{2}}{M^{2}}\right)\right. \\
& \left.+\frac{1}{2}\left(\tau^{2}+(1-\tau)^{2}\right) \ln \left(\frac{(1-\tau)^{2}}{\tau}\right)+\frac{1}{4}(1-\tau)(7 \tau-3)\right] .
\end{aligned}
$$

Note that both the quark and gluon initiated processes, after the mass factorisation, are free of any IR divergences. We use these results for our further analysis after folding with appropriate parton distributions. For the numerical calculation we made the renormalisation scale $\mu$ and the factorisation scale $M$ equal (i.e. $\mu=M=\mu_{F}$ ).

\section{$5 \quad$ Results and Discussion}

Having obtained the analytic expressions in the last section, we now endeavour to see the numerical size of these corrections. To this end, one needs to define the leadingorder and the next-to-leading-order cross-sections for a $p \bar{p}$ (or equivalently $p p$ ) collider:

$\sigma_{\mathrm{LO}}$ : convolute the cross-section of eqn.(7) with the appropriate LO quark distributions;

$\sigma_{\mathrm{NLO}}:$ convolute the cross-section of eqns.(38,45) with the appropriate NLO quark and gluon distributions. 
Of course an additional factor of $1 / 2$ needs to be included in the cross-sections to account for the chiral nature of the $R_{\mathrm{p}}$ interactions.

Before we start in earnest, a minor digression. Since QCD corrections are flavourblind, the value of the coupling $\lambda^{\prime}$ is immaterial and only serves to set an overall scale for the cross-section. To be concrete, we shall choose to work with

$$
\lambda^{\prime}=0.01
$$

irrespective of flavour and the mass of the sneutrino/slepton. While this may seem to be an inconsistent choice for a light $\tilde{e}$ or $\tilde{\nu}_{e}$ (see Table 1), this is not quite germane to the issue at hand.

\subsection{Sneutrino Production}

To begin with, we concentrate on the resonance production of a sneutrino starting with a $d \bar{d}$ initial state (at the Born level). In Fig.1, we plot both the LO and the NLO crosssections as a function of the sneutrino mass and for three different choices of parton distributions. The renormalisation scale $\mu_{F}$ as described in the previous section has been chosen to be the same as the sneutrino mass.

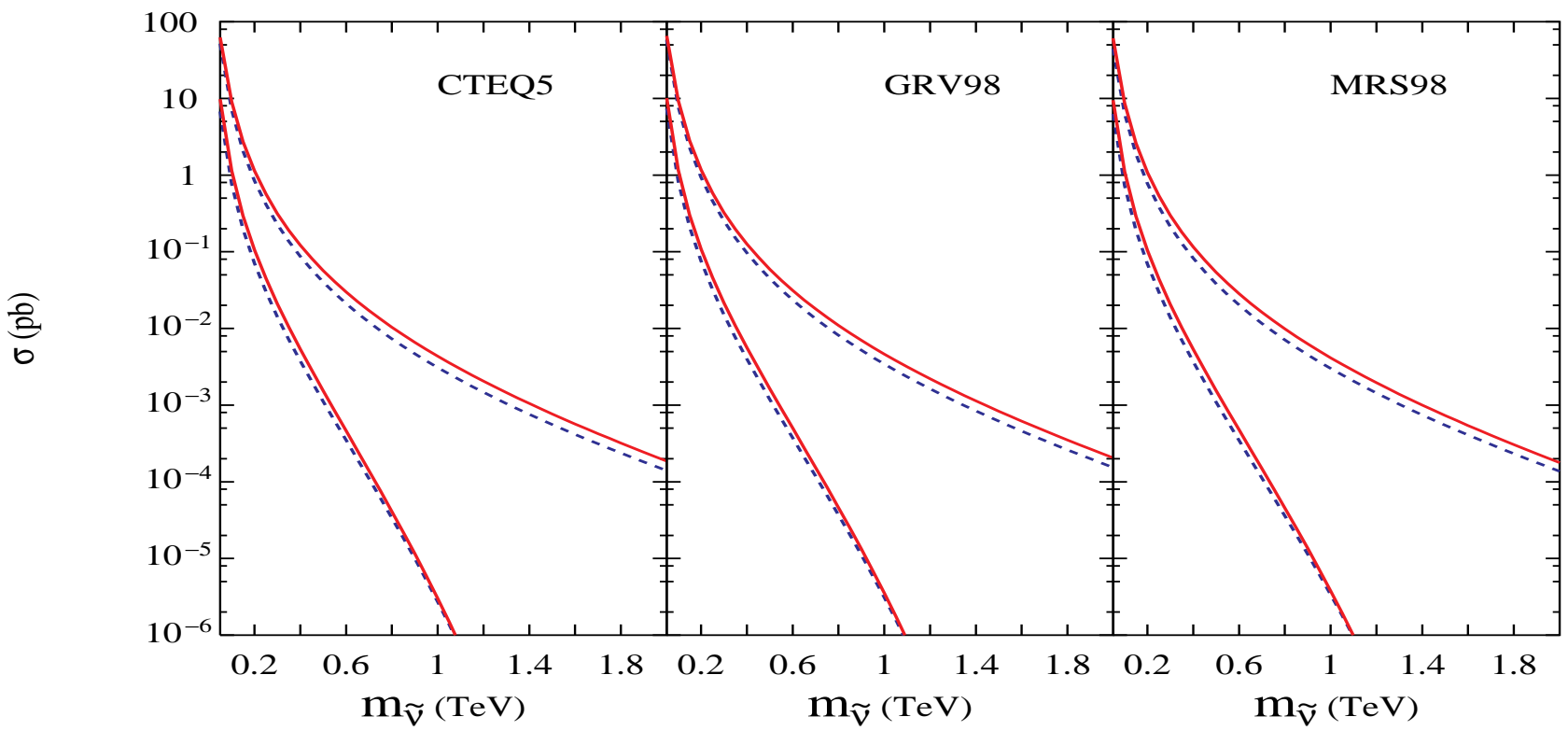

Figure 1: Cross-section for resonant sneutrino production at the Tevatron (lower curves) and at the LHC (upper curves). The solid (dashed) curves represent the NLO (LO) cross-sections. The $R$-parity violating coupling $\lambda_{i 11}^{\prime}$ has been set to 0.01. The three cases correspond to structure function parametrisations CTEQ5, GRV98 and MRS98 respectively.

On the face of it, the three sets of curves look quite similar, a point that we shall remark on later. The dependence on the scalar mass is as expected and is occasioned by both 
the fall in the parton-level cross-sections and the rapid decrease of the parton densities at high momentum fractions. The latter effect, understandably, is more pronounced at the Tevatron than at the LHC.

To parametrise the effect of the NLO corrections, it is common to introduce the $K$ factor:

$$
K \equiv \frac{\sigma_{\mathrm{NLO}}}{\sigma_{\mathrm{LO}}}
$$

which we plot in Fig. . Let us concentrate first on the results for the Tevatron. The near monotonic decrease of $K$ with $m_{\tilde{\nu}}$ is not unexpected. As $m_{\tilde{\nu}}$ increases, we are sampling increasingly larger values of parton momenta. This has two immediate consequences. For one, the Compton contribution becomes increasingly irrelevant. But more importantly, a large $m_{\tilde{\nu}}$ also means that the 'primary quark' is less able to radiate off a gluon. In fact, as $m_{\tilde{\nu}} \rightarrow \sqrt{s_{p \bar{p}}} / 2, K$ approaches unity.

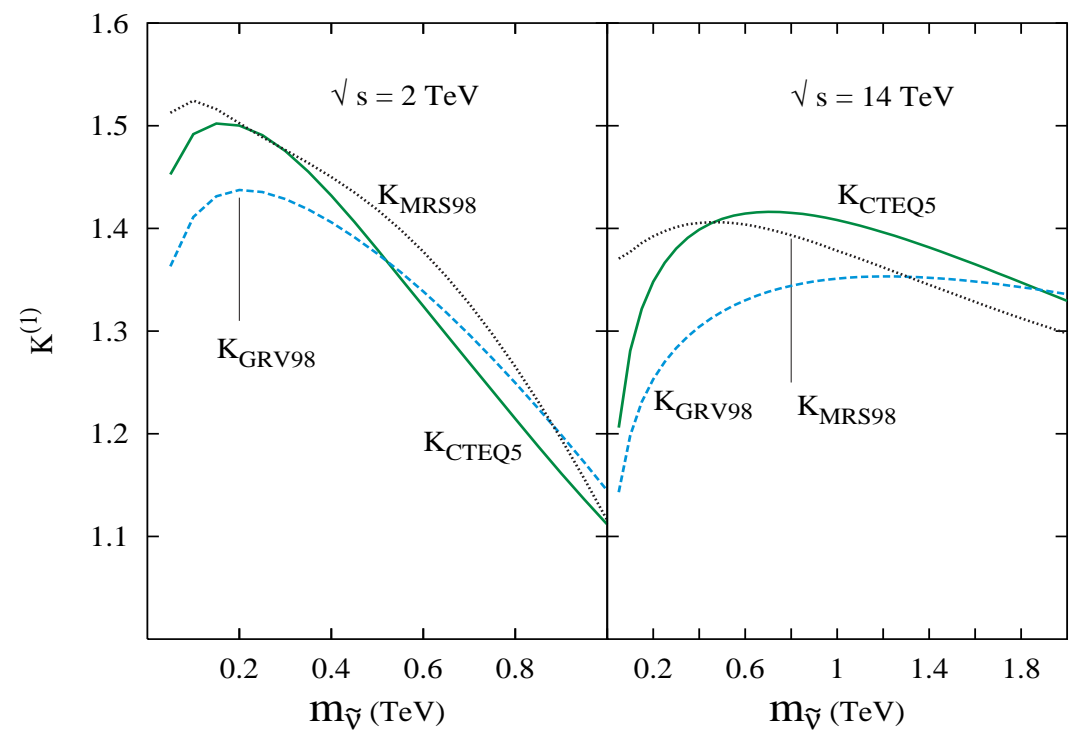

Figure 2: The $K$ factor for the process involving coupling $\lambda_{i 11}^{\prime}$ as a function of the sneutrino mass as calculated for different parton distributions. The two graphs correspond to the Tevatron and the $\mathrm{LHC}$ respectively.

It might seem significant that the $K$-factors, as calculated with different sets of parton distributions, vary significantly amongst themselves. This only reflects the dependence of the cross-sections on our ansätz for the parton densities. This assertion is supported by the fact the differences are more pronounced for smaller sneutrino masses, where the cross-section receives a larger contribution from relatively low momenta $\left(x_{\mathrm{Bj}}\right)$ partons and hence prone to larger parametrisation errors. Interestingly, the difference in the $K$-factors arise, in a large measure, due to the differences in the LO cross-sections. The relative differences between the cross-sections, as calculated with the different Ansätze, actually decrease as we go from the LO to the NLO calculations, and can be expected to become smaller as progressively higher order corrections are incorporated.

Turning now to the results for the LHC, we see that, for $m_{\tilde{\nu}} \gtrsim 300 \mathrm{GeV}$, the behaviour is quite analogous to the case of the Tevatron. Although the fall with the sneutrino 
mass seems to be slower, it should be remembered that the graph covers a much smaller range in $m_{\tilde{\nu}} / \sqrt{s_{p p}}$. The behaviour at small masses $(\$ 300 \mathrm{GeV})$ seems puzzling though. However, one must realize that the cross-section for such light sneutrinos is dominated by low momenta partons. And since existing data does not probe the parton densities unto very low $x_{\mathrm{Bj}}$, the various Ansätze naturally have differing predictions. Although it does not show up in the curves of Fig.1, again the difference in the $K$-factor is dominated by the deviations in the LO cross-sections rather than the NLO ones.

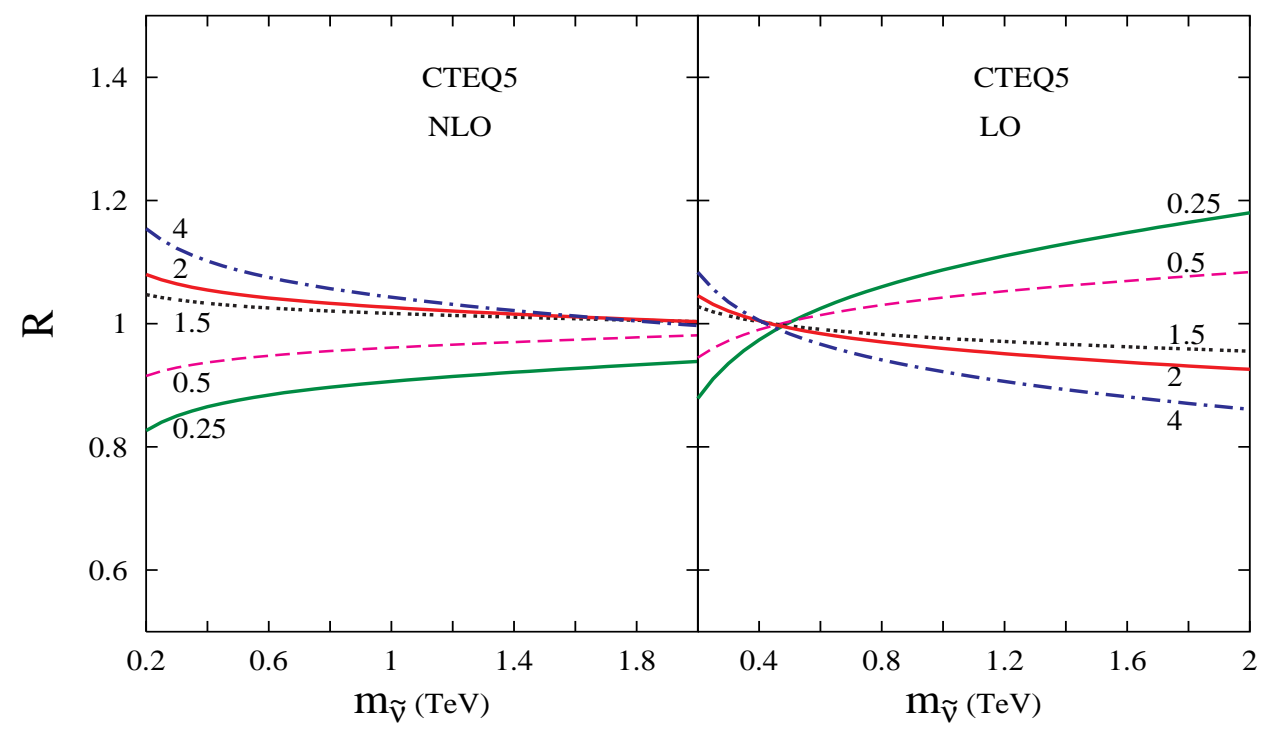

Figure 3: The dependence of the cross-sections at the LHC on the value of the factorisation scale $\mu_{F}$. The ratio $R$ (see eqn. $(\sqrt{47})$ ) compares the cross section to the reference point of $\mu_{F}=m_{\tilde{\nu}}$. The legends on the graphs correspond to the ratio $\mu_{F} / m_{\tilde{\nu}}$. The left and right panels correspond to the NLO and LO cross-sections respectively. The CTEQ5 densities have been used.

Having explored the dependence of the $K$-factor on the sneutrino mass and the choice of parton densities, we now turn to the final 'unknown' viz. the renormalisation scale. Although the most natural scale is that of the sneutrino mass (with many other similar analyses making this choice too), the exact value of $\mu_{F}$ is somewhat ambiguous. To quantify the ensuing dependence, we define the ratio

$$
R_{\mathrm{NLO}}\left(\mu_{F} ; m_{\tilde{\nu}}\right) \equiv \frac{\sigma_{\mathrm{NLO}}\left(\mu_{F}\right)}{\sigma_{\mathrm{NLO}}\left(\mu_{F}=m_{\tilde{\nu}}\right)}
$$

operative within a given parton density parametrisation. An analogous expression can also be defined for the LO cross sections. In Fig. 3, we exhibit the variation of $R\left(\mu_{F} ; m_{\tilde{\nu}}\right)$ for both the LO and the NLO cross sections. As the graph shows, the variation of the cross-section with $\mu_{F}$ is relatively small. Furthermore, the variation reduces as one progresses from the LO calculation to the NLO. The last observation lends hope that the remaining scale ambiguity can, presumably, be reduced by adding still higher order corrections.

\footnotetext{
${ }^{4}$ While we show the dependence only for the CTEQ parametrization, those for the other parametrizations are very similar.
} 


\subsubsection{Initial states with strange quarks}

We now consider other possibilities for resonance production. Restricting ourselves to sneutrinos for the moment, note that a non-zero value for some of the other $\lambda^{\prime}$ couplings could lead to alternate tree-level processes such as:

$$
\begin{array}{rll}
\lambda_{i 22}^{\prime} & : & s+\bar{s} \rightarrow \tilde{\nu}_{i}, \\
\lambda_{i 12}^{\prime} \text { or } \lambda_{i 21}^{\prime} & : & d+\bar{s} \rightarrow \tilde{\nu}_{i} .
\end{array}
$$

Although the chirality structure of the interaction term is different for the two cases in the second line, it is of no consequence for either the LO cross-section or the NLO corrections. In Fig. 目, we plot these cross-sections for foth the Tevatron and the LHC. For an identical value of the $\mathrm{R}_{\mathrm{p}}$ violating coupling, the total cross-section is much smaller than that in Fig.11. This is only to be expected as the strange-quark is a part of the sea and consequently its flux is much smaller than that for the $d$-quark. Thus, for the LHC, we have the relation $\sigma(d \bar{d})>\sigma(d \bar{s})>\sigma(s \bar{d})>\sigma(s \bar{s})$, while for the Tevatron, the second inequality is replaced by an equality.

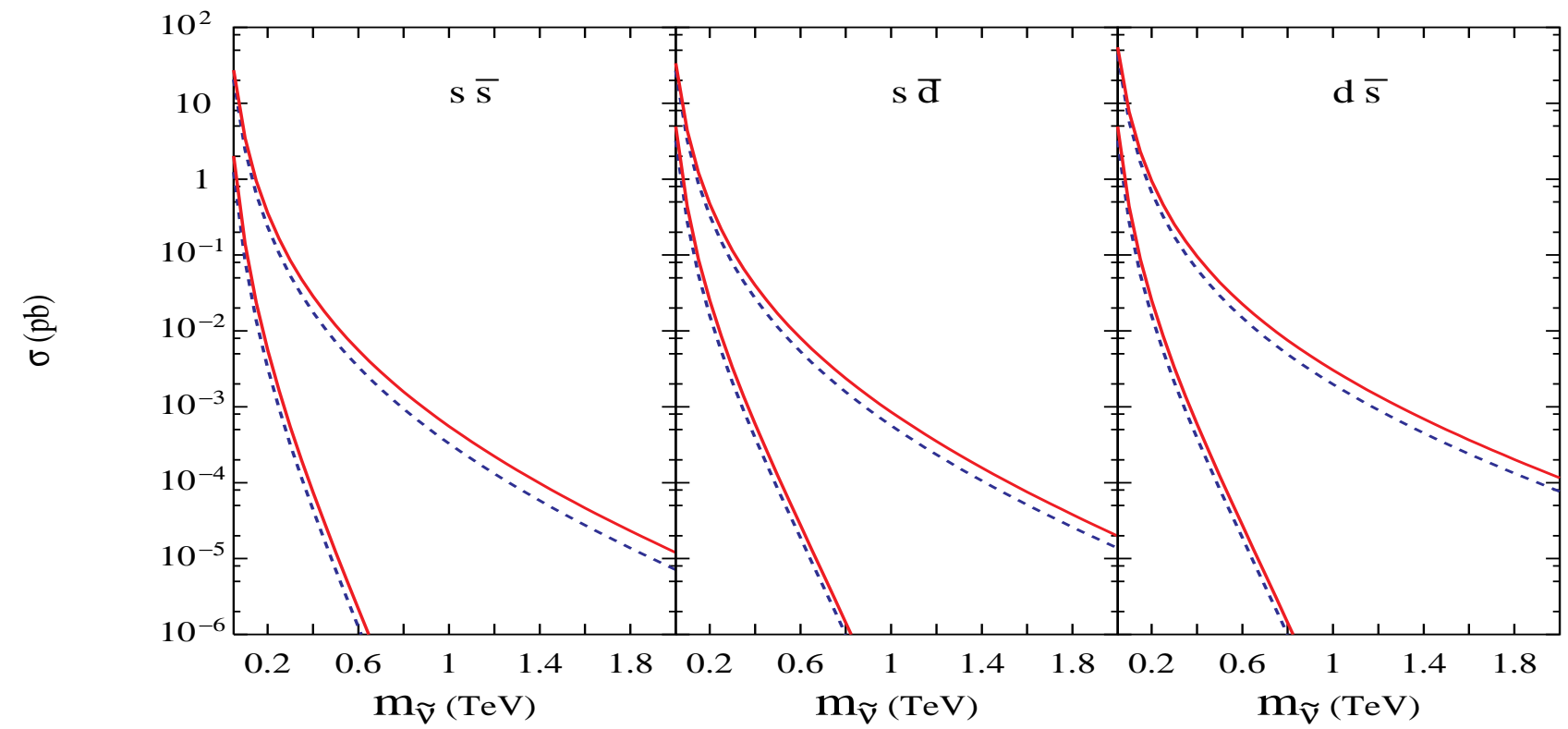

Figure 4: Cross-section for resonant sneutrino production at the Tevatron (lower set of curves) and LHC (upper set). For each set, the solid (dashed) refer to $N L O(L O)$ cross sections. The respective Born-level initial states are indicated in each panel. The value of the R-parity violating coupling (see eqn.(48)) has been set to be 0.01 and the CTEQ5 parametrisation has been used.

As in the previous case, we may once again choose to parametrise the NLO corrections in the form of a $K$-factor. And, although we have chosen to present the cross-sections only for the CTEQ5 parton distributions, it is quite instructive to consider the dependence on the parametrisation. In Fig.5, we present this for the $s \bar{s}$ initial state. The

\footnotetext{
${ }^{5}$ Of course, similar production mechanisms involving a $b$-quark in the initial state is possible too. However, owing to the small flux for the $b$-quark, these are not of much interest phenomenologically.
} 
wide difference between the $K$-factor as calculated within CTEQ5 [29], with those obtained in the context of MRS98 [30] or GRV98 [31] may seem to be a matter of concern. Interestingly, unlike in the case of the $d \bar{d}$ initial state, the difference in $K$ here cannot be ascribed to the LO parton distributions. Rather, the blame lies on the NLO parton distributions, in particular the much larger strange-quark flux in the CTEQ5 parametrisation (as compared to GRV98 or MRS98). Although this large deviation is partly offset by a sizable negative contribution from the Compton diagram, the latter effect is clearly subdominant. While such a discrepancy might seem vexing, it is easier to appreciate once one considers the experimental inputs in the parton density parametrisations, especially in the large $x$ region. For example, the CTEQ collaboration uses jet measurement data whereas MRS used prompt photon data. And since our curves for the Tevatron reach unto a much larger $x$ value than those for the LHC, the difference is more pronounced in the former case. Notwithstanding this post-facto rationalisation, the resultant $K_{\mathrm{CTEQ5}}$ remains uncomfortably large, and moreover, does not approach unity as $m_{\tilde{\nu}} \rightarrow \sqrt{s_{p \bar{p}}}$. This is symptomatic of an inherent problem in the CTEQ5 parametrisation for the heavier sea quark distributions.

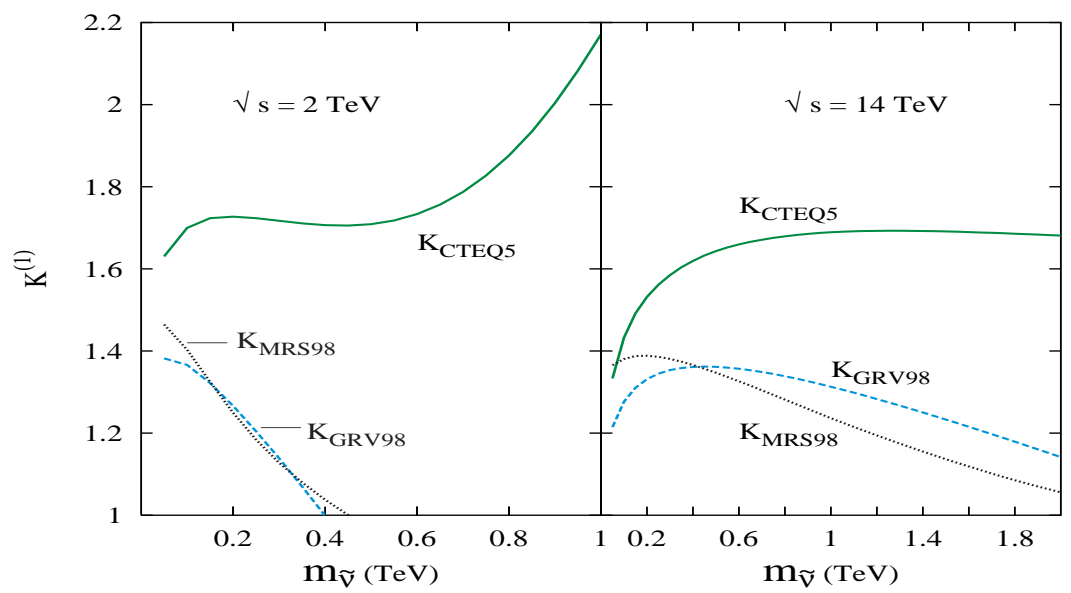

Figure 5: The $K$ factor for the processes with coupling $\lambda_{i 22}^{\prime}$ at the Tevatron as a function of the slepton (sneutrino) mass.

The same problem is also reflected in the $K$-factors for the two other cases of eqn.(48) namely those with $s \bar{d}$ and $d \bar{s}$ initial states. Once again $K_{\mathrm{MRS} 98}$ and $K_{\mathrm{GRV} 98}$ are quite similar while $K_{\mathrm{CTEQ5}}$ is significantly different (see Fig.6).

\subsection{Charged slepton production}

We finally consider slepton production. Governed by eqn.(2), the relevant piece of the interaction Lagrangian is readily seen to have the same structure as the piece responsible for sneutrino production. Once again, various combinations of quark-anti-quark pair could feature in the production process. However, for brevity's sake we shall confine ourselves to a discussion of only the processes corresponding initiated, at the Born level, by $u \bar{d}$ (and 


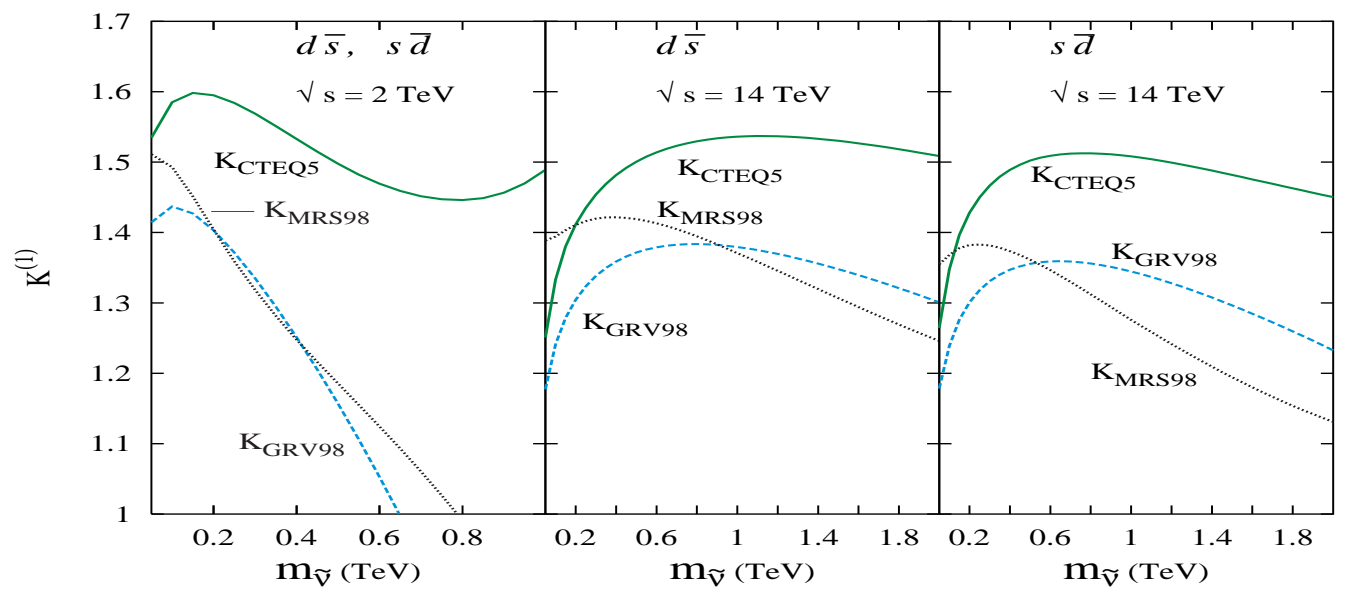

Figure 6: The $K$ factor for the processes with coupling $\lambda_{i 12}^{\prime}$ or $\lambda_{i 21}^{\prime}$ at the Tevatron as a function of the slepton (sneutrino) mass.

$d \bar{u})$. Of course, in the context of the Tevatron, the two cross-sections would be identical, whereas in the LHC, the former would be slightly larger.

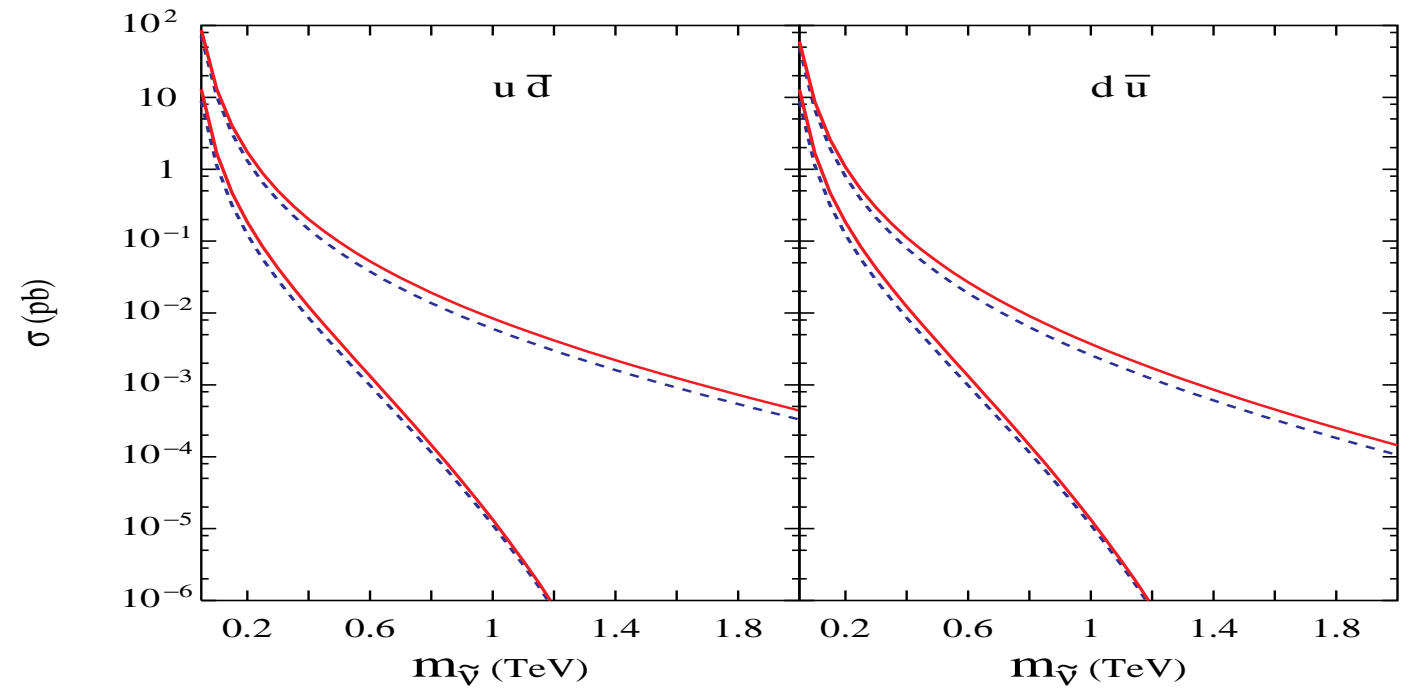

Figure 7: Cross-section for charged slepton production at the Tevatron (lower set of curves) and LHC (upper set). For each set, the solid (dashed) refer to $N L O(L O)$ cross sections. The respective Born-level processes are given by $d+\bar{u} \rightarrow \tilde{\ell}^{-}$and $u+\bar{d} \rightarrow \tilde{\ell}^{+}$. The value of the $R$-parity violating coupling has been set to 0.01 and the CTEQ5 parametrisation has been used.

As Fig.7 demonstrates, the behaviour of the cross-section is identical to that of sneutrino production in $d \bar{d}$ collision (although the magnitude is somewhat larger). This was only to be expected as the flux of the $u$-quark inside the proton is similar to that for the $d$-quark. To be very precise, the valence $u$-density is a bit higher than the valence $d$, whereas the sea-densities are very similar for the two. Consequently, we should expect the behaviour of the $K$-factor to be quite similar again, as is borne out by Fig.8. The remarkable similarity between $K_{u \bar{d}}$ (and hence $K_{d \bar{u}}$ too for the Tevatron case) and $K_{d \bar{d}}$ 
(Fig.2) is a testimonial to the fact that the only difference between the two cases arises from the small effect due to isospin violation in the valence quark densities (which, of course manifests itself primarily in the large scalar mass region).

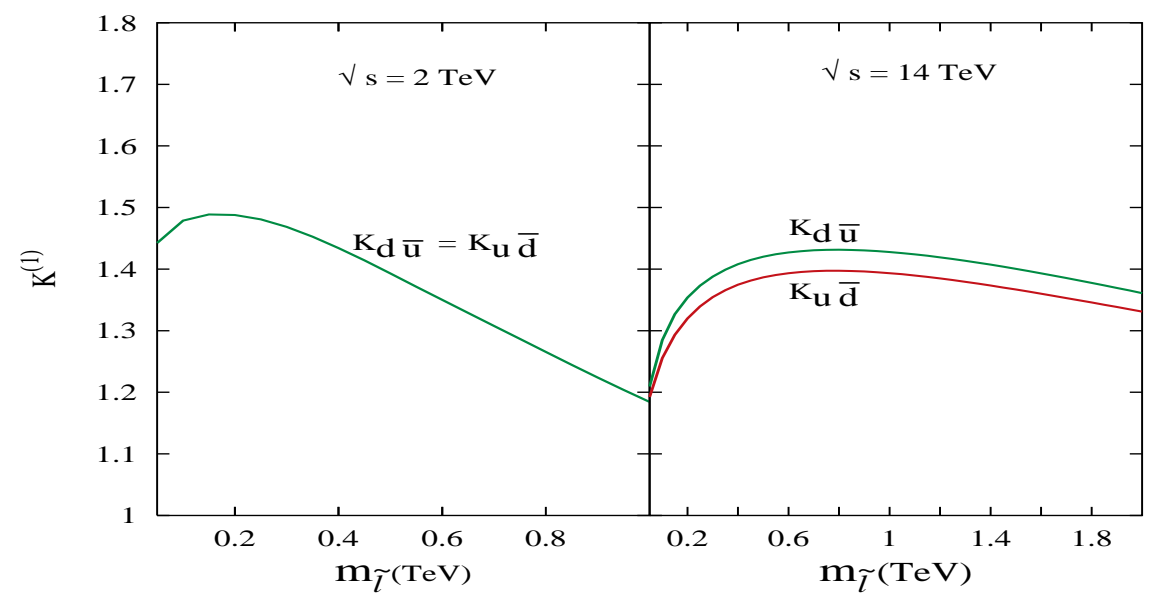

Figure 8: The $K$ factor for charged slepton production (processes of Fig. (7) at the Tevatron as well as at the LHC as a function of the slepton mass. The CTEQ5 densities have been used.

\section{Conclusions}

To summarise, we have calculated the NLO corrections to the resonant sneutrino and slepton production cross-sections (within $R_{\mathrm{p}}-\mathrm{MSSM}$ ) in the context of Tevatron and LHC. We find that for processes controlled, at the Born level, by first-generation quarks, the ensuing $K$-factor is a fairly sensitive function of the scalar mass. For masses below about $1 \mathrm{TeV}$, the correction can vary between $10 \%$ and $50 \%$ at the Tevatron with the correction falling steeply for higher masses. At the LHC, the mass-dependence is reduced significantly, and for masses less than $2 \mathrm{TeV}$, varies only between 1.2 and 1.45. While there is a significant dependence on the structure function used, the effect is much less pronounced for the NLO calculation than for the LO. This lends us hope that once the next order effects are incorporated the theoretical ambiguity would reduce to insignificant levels.

For production processes involving quarks of higher generations, the situation is not so simple. The $K$-factors could be much larger, and worse, show a marked dependence on the particular density parametrisation used. This is but a reflection of the fact that these distributions are known with much less precision and hence vary significantly between parametrisations. Since the production cross-sections themselves are large enough to be interesting, an NNLO calculation thus seems to be called for.

Finally, the calculations presented in this paper are not particular to supersymmetric theories, but can be applied to any color-singlet scalar (pseudoscalar) coupling to a quarkanti-quark pair. 


\section{Acknowledgements}

We would like to thank Anindya Datta for many useful discussions and, more particularly, for his participation during the early stages of the project. DC and VR would like to thank the Theory Division, CERN for hospitality while part of the project was being carried out. DC also acknowledges the Department of Science and Technology, India for financial assistance under the Swarnajayanti Fellowship grant.

\section{References}

[1] LEP Electroweak Working Group, LEPEWWG/2001-01.

[2] H.P. Nilles, Phys. Rep. 110 (1989) 1;

H.E. Haber and G.L. Kane, Phys. Rep. 117 (1985) 75;

S. Dawson, Nucl. Phys. B261 (1985) 297.

[3] J.C. Pati and A. Salam, Phys. Rev.D10 (1974) 275.

H. Georgi and S.L. Glashow, Phys. Rev. Lett. 32 (1974) 438;

For reviews, see P. Langacker, Phys. Rep. 72 (1981) 185.

[4] P. Frampton, Phys. Rev.D42 (1990) 3892;

P. B. Pal and U. Sarkar, Phys. Rev.D49 (1994) 3721.

[5] S. Dimopoulos et al., Phys. Rev. D41, 2099 (1990);

H. Dreiner and G. G. Ross, Nucl. Phys. B365, 597 (1991);

B. C. Allanach et al., hep-ph/9906224;

H. Dreiner, P. Richardson, and M. H. Seymour, JHEP 0004, 008 (2000), hepph/9912407.

[6] J. Kalinowski et al., Phys. Lett. B414, 297 (1997) [hep-ph/9708272;

J. L. Hewett and T. G. Rizzo, hep-ph/9809525;

H. Dreiner, P. Richardson, and M. H. Seymour, hep-ph/9903419; hep-ph/0001224;

G. Moreau, M. Chemtob, F. Deliot, C. Royon, and E. Perez, Phys.Lett. B475, 184 (2000) hep-ph/9910341;

G. Moreau, E. Perez, and G. Polesello, hep-ph/0002130; Nucl.Phys. B604,3 (2001) hep-ph/0003012];

S. Abdullin et al., hep-ph/0005142.

[7] A. Datta et al., Phys. Rev. D56, 3107 (1997) hep-ph/9704257;

J.-M. Yang et al., hep-ph/9802305;

R. J. Oakes et al., Phys. Rev. D57, 534 (1998) hep-ph/9707477;

E. L. Berger, B. W. Harris, and Z. Sullivan, Phys. Rev. Lett. 83, 4472 (1999) [hepph/9903549].

[8] S. Dimopoulos and L. J. Hall, Phys. Lett. B207, 210 (1988);

G. F. Giudice et al., hep-ph/9602207; 
ECFA/DESY LC Physics Working Group, E. Accomando et al., Phys. Rept. 299, 1 (1998) hep-ph/9705442;

J. Erler, J.L. Feng, and N. Polonsky, Phys. Rev. Lett. 78, 3063 (1997) thepph/9612397;

J. Kalinowski, R. Rückl, H. Spiesberger, and P. M. Zerwas, Phys. Lett. B406, 314 (1997) hep-ph/9703436.

[9] V. Barger, G. F. Giudice, and T. Han, Phys. Rev. D40, 2987 (1989).

[10] J.L. Hewett, Snowmass Summer Study (1990);

J. Butterworth and H. Dreiner, Nucl. Phys. B397, 3 (1993) hep-ph/9211204;

D. Choudhury and S. Raychaudhuri, Phys. Lett. B401 (1997) 54 [hep-ph/9702392;

G. Altarelli et al., Nucl. Phys. B506 (1997) 3 hep-ph/9703276;

J. Kalinowski et al., Z. Phys. C74 (1997) 595 hep-ph/9703288;

T. Kon and T. Kobayashi, Phys. Lett. B409 (1997) 265 hep-ph/9704221;;

G. Altarelli, G.F. Giudice, and M.L. Mangano, Nucl. Phys. B506 (1997) 29 [hepph/9705287];

J. Ellis, S. Lola, and K. Sridhar, Phys. Lett. B408 (1997) 252 hep-ph/9705416];

M. Carena et al., Phys. Lett. B414 (1997) 92 hep-ph/9707458.

[11] Z.Kunszt, W.J.Stirling, Z. Phys. C75 (1997) 453;

T.Plehn et al., Z. Phys. C74 (1997) 611.

[12] Y. Fukuda et al. (Super-Kamiokande collab.), Phys. Rev. Lett. 81 (1998) 1562; Phys. Lett. B436 (1998) 33.

[13] B. Armbruster et al.(KARMEN collab.), Phys. Lett. B348 (1995) 19;

D. Choudhury and S. Sarkar, Phys. Lett. B401 (1996) 87;

B. Zeitnitz, talk given at NEUTRINO'98, Takayama (1998);

R. Maschuw, talk given at WIN'99, Cape Town (1999);

D. Choudhury, H. Dreiner, P. Richardson and S. Sarkar, Phys. Rev.D61 (2000) 095009.

[14] P. Fayet, Phys. Lett. B69 (1977) 489;

G. Farrar and P. Fayet, Phys. Lett. B76 (1978) 575.

[15] L. E. Ibanez and G. G. Ross, Nucl. Phys. B368 (1992) 3.

[16] L. J. Hall and M. Suzuki, Nucl. Phys. B231, 419 (1984).

[17] A. Bouquet and P. Salati, Nucl. Phys. B284, 557 (1987);

A.E. Nelson and S.M. Barr, Phys. Lett. B246, 141 (1990);

E. Roulet and D. Tommasini, Phys. Lett. B256, 218 (1991);

B.A. Campbell, S. Davidson, J. Ellis and K. Olive, Phys. Lett. B256, 457 (1991);

W. Fischler, G. Giudice, R.G. Leigh and S. Paban, Phys. Lett. B258, 45 (1991).

[18] H. Dreiner and G.G. Ross, Nucl. Phys. B410, 188 (1993) hep-ph/9207221. 
[19] D.K. Ghosh, S. Raychaudhuri and K. Sridhar, Phys. Lett. B396 (1997) 197 hepph/9608352];

K. Hikasa, J. M. Yang and B. Young, Phys. Rev.D60 (1999) 114041;

D. Choudhury, Phys. Lett. B346 (1995) 291 hep-ph/9408250;

A. Datta, Phys. Rev.D65 (2002) 054019 [hep-ph/0108150];

G. Bhattacharyya, D. Choudhury and K. Sridhar, Phys. Lett. B349 (1995) 118 [hepph/9412259];

D. Choudhury, R.M. Godbole and G. Polesello, hep-ph/0207248.

[20] B. Abbott et al.(D0 Collab.), Phys. Rev. Lett. 83 (1999) 4476 hep-ex/9907019; Phys. Rev. Lett. 84 (2000) 2088.

[21] F. Abe et al.(CDF Collab.), Phys. Rev. Lett. 83 (1999) 2133 hep-ex/9908063;

D. Choudhury and S. Raychaudhuri, Phys. Rev.D56 (1997) 1778 [hep-ph/9703369.

[22] K. Agashe and M. Graesser, Phys. Rev.D54 (1995) 4445 [hep-ph/9510439;

D. Choudhury and P. Roy, Phys. Lett. B378 (1996) 153 hep-ph/9603363;

F. Vissani and A.Yu. Smirnov, Phys. Lett. B380 (1996) 317 hep-ph/9601387.

[23] G. Bhattacharyya, Nucl. Phys. B (Proc. Suppl.) 52A (1996) 83;

H. Dreiner, (electronic archive: hep-ph/9707435 v2);

R. Barbier et al., (electronic archive: hep-ph/9810232).

[24] H. Klapdor-Kleingrothaus et al., Prog. Part. Nucl. Phys. 32 (1994) 261;

M. Hirsch, H. V. Klapdor-Kleingrothaus, and S. G. Kovalenko, Phys. Rev. Lett. 75, 17 (1995); Phys. Rev. D53, 1329 (1996);

K. S. Babu and R. N. Mohapatra, Phys. Rev. Lett. 75 (1995) 2276 hep-ph/9506354;

J. W. F. Valle, hep-ph/9509306.

[25] A.I. Belesev et al., Phys. Lett. B350 (1995) 263;

C. Weinheimer et al., Phys. Lett. B300 (1993) 210.

[26] G. Bhattacharyya and D. Choudhury, Mod. Phys. Lett. A10 (1995) 1699 hepph/9503263.

[27] A. S. Joshipura, V. Ravindran and S. K. Vempati, Phys. Lett. B451, 98 (1999) hep-ph/9706482.

[28] S. Davidson, D. Bailey and B. Campbell Z. Phys. C61 (1994) 613 hep-ph/9309310;

C.S. Wood, Science 279 (1997) 1759;

W.J. Marciano and J.L. Rosner, Phys. Rev. Lett. 65 (1990) 2963;

W.J. Marciano, 1997 INT Summer Workshop.

[29] H.L. Lai et al., Eur. Phys. J. C12, 375 (2000) hep-ph/9903282.

[30] A.D. Martin, et al., Eur. Phys. J. C4, 463 (1998) hep-ph/9803445.

[31] M. Gluck, E. Reya and A. Vogt, Eur. Phys. J. C5, 461 (1998) hep-ph/9806404. 